\title{
SYNTHESIS, CHARACTERIZATION AND BIOLOGICAL EVALUATION OF A GLYCINE-RICH PEPTIDE - CHERIMOLACYCLOPEPTIDE E
}

\author{
RAJIV DAHIYA* \\ Institution where research was carried out \\ Department of Pharmaceutical Chemistry, Rajiv Academy for Pharmacy, Mathura 281001 (UP), India.
}

(Received $28^{\text {th }}$ February 2007 - Accepted $15^{\text {th }}$ May 2007)

\begin{abstract}
The synthesis of a natural cyclic peptide cherimolacyclopeptide E (12) by coupling of tripeptide units Boc-gly-leu-gly-OCH $\mathbf{O C}_{3}(\mathbf{9})$ and Boc-phe-tyr-pro-OCH $_{3}$ (10) after proper deprotection at carboxyl and amino terminals followed by cyclization of linear hexapeptide segment, is described. Structure elucidation of the cyclopeptide 12 is based on detailed spectral analysis such as FTIR, ${ }^{1} \mathrm{H}$ NMR, ${ }^{13} \mathrm{C}$ NMR, FAB MS and elemental analysis. After biological screening, the newly synthesized peptide exhibited high cytotoxicity against Dalton's lymphoma ascites (DLA) and Ehrlich's ascites carcinoma (EAC) cell lines with CTC ${ }_{50}$ values of 2.76 and $4.96 \mu \mathrm{M}$, and potent antimicrobial activity against pathogenic microbes P. aeruginosa, E.coli and C. albicans with MICs between 12.5-6 $\mu \mathrm{g} / \mathrm{mL}$. Furthermore, compound 12 possessed moderate anthelmintic activity against earthworms M. konkanensis, P. corethruses and Eudrilus sp. at $2 \mathrm{mg} / \mathrm{mL}$ dose level.
\end{abstract}

Keywords: cherimolacyclopeptide E, cyclic hexapeptide, cytotoxicity, antimicrobial evaluation, anthelmintic activity

\section{INTRODUCTION}

During past decades, plants have well proved their potential to produce a wide spectrum of natural products with interesting bioactivities ${ }^{1-6}$. Among these, cyclic peptides and related congeners with unique structures and wide pharmacological profile ${ }^{7-10}$ have received special attention which may prove better candidates to overcome the problem of wide spread increase of resistance towards conventional drugs. Different pharmacological activities possessed by cyclic peptides obtained from latex, seeds and roots of plants, include antitumour activity ${ }^{11-13}$, immunosuppressive and antimalarial activity ${ }^{14}$ vasorelaxant activity ${ }^{15,16}$, tyrosinase inhibitory activity ${ }^{17,18}$ and estrogen-like activity $^{19,20}$. Recently, a natural cyclopolypeptide, cherimolacyclopeptide E, has been isolated from seeds of small tree Annona cherimola and the structure was elucidated by MS/MS fragmentation experiments, extensive 2D-NMR analysis/chemical degradation and showed significant cytotoxic activity against KB (human nasopharyngeal carcinoma) cells ${ }^{21}$.

As part of our ongoing efforts on synthetic aspects of bioactive cyclic peptides ${ }^{22-26}$, the present study was aimed at synthesis of novel cyclic hexapeptide - cherimolacyclopeptide E 12. Keeping in view of significant bioactivities possessed by various cyclopeptides ${ }^{27,28}$, the above synthetic peptide was further subjected to antibacterial, antifungal, anthelmintic and cytotoxic activity studies.

\section{EXPERIMENTAL}

\section{GENERAL}

All the reactions requiring anhydrous conditions were conducted in flame dried apparatus. Melting point was determined by open capillary method and is uncorrected. L-amino acids, dicyclohexylcarbodiimide (DCC), trifluoroacetic acid (TFA), p-nitrophenol (pnp), N-methylmorpholine (NMM), triethylamine (TEA), di-tert-butylpyrocarbonate $\left(\right.$ Boc $\left._{2} \mathrm{O}\right)$ and pyridine $\left(\mathrm{C}_{5} \mathrm{H}_{5} \mathrm{~N}\right)$ were obtained from Spectrochem Limited (Mumbai, India). IR spectra were recorded on Shimadzu 8700 FTIR spectrophotometer (Shimadzu, Japan) using a thin film supported on $\mathrm{KBr}$ pellets for solids and $\mathrm{CHCl}_{3}$ as solvent for intermediate semisolids (in $\mathrm{cm}^{-1}$ ). ${ }^{1} \mathrm{H}$ NMR and ${ }^{13} \mathrm{C}$ NMR spectra were recorded on Bruker AC NMR spectrometer ( $300 \mathrm{MHz}$ ), (Brucker, USA) using $\mathrm{CDCl}_{3}$ as solvent and tetramethylsilane as internal standard (in ppm). Mass spectra was recorded on JMS-DX 303 Mass spectrometer (Jeol, Tokyo, Japan) operating at $70 \mathrm{eV}$ using fast atom bombardment technique. Elemental analyses of all compounds were performed on Vario EL III elemental analyzer (Elementar, Germany). Optical rotation of the peptides was measured on automatic polarimeter (Optics Tech, Ghaziabad, India) in a $2 \mathrm{dm}$ tube at $25^{\circ} \mathrm{C}$ using sodium lamp and methanol as solvent. Purity of synthesized cyclopeptide as well as intermediates was checked by TLC on precoated silica gel $\mathrm{G}$ plates utilizing $\mathrm{CHCl}_{3} / \mathrm{CH}_{3} \mathrm{OH}$ as developing solvent in different ratios $(9: 1 / 7: 3 \mathrm{v} / \mathrm{v})$ and brown spots were detected on exposure to iodine vapours in a tightly closed chamber.
General procedure for synthesis of Boc-amino acids $(1,2)$

L-amino acid $(20 \mathrm{mmol})$ was dissolved in $1 \mathrm{~N} \mathrm{NaOH}(20 \mathrm{~mL})$ and isopropanol $(20 \mathrm{~mL})$. Di-tert-butylpyrocarbonate $(6 \mathrm{~mL}, 26 \mathrm{mmol})$ in isopropanol $(10 \mathrm{~mL})$ was added followed by $1 \mathrm{~N} \mathrm{NaOH}(20 \mathrm{~mL})$ to the resulting solution. The solution was stirred at RT for $2 \mathrm{~h}$, washed with light petroleum ether (bp $\left.40-60{ }^{\circ} \mathrm{C}\right)(20 \mathrm{~mL})$, acidified to $\mathrm{pH} 3.0$ with $2 \mathrm{~N} \mathrm{H}_{2} \mathrm{SO}_{4}$ and finally extracted with chloroform $(3 \times 20 \mathrm{~mL})$. The organic layer was dried over anhydrous $\mathrm{Na}_{2} \mathrm{SO}_{4}$ and evaporated under reduced pressure to give the crude product which was finally crystallized from chloroform and petroleum ether (bp $40-60{ }^{\circ} \mathrm{C}$ ).

\section{tert-Butyloxycarbonyl-glycine (1).}

This compound was obtained according to the general procedure as white crystals, mp $93{ }^{\circ} \mathrm{C}$, yield $83 \%,[\alpha]_{\mathrm{D}}+2.7^{\circ}, \mathrm{R}_{\mathrm{f}}-0.61$; IR: $3292-2488(\mathrm{~m} / \mathrm{br}, \mathrm{OH}$ str, $\mathrm{COOH}$ ); 3133 (m, NH str, amide); 2927, 2850 (m, CH str, asym and sym, $\mathrm{CH}_{2}$ ); 1712 (s, $\mathrm{C}=\mathrm{O}$ str, $\mathrm{COOH}$ ); 1635 (s, $\mathrm{C}=\mathrm{O}$ str, amide); 1531 (m, NH bend, amide); 1466 ( $\mathrm{m}, \mathrm{CH}$ bend (scissoring), $\mathrm{CH}_{2}$ ); 1387, 1362 (m, $\mathrm{CH}$ bend, butylt); 929 (w, $\mathrm{CH}_{3}$ rocking, butyl-t). ${ }^{1} \mathrm{H}$ NMR: 10.02 (br. s, $1 \mathrm{H}, \mathrm{OH}$ ); 6.04 (br. s, $1 \mathrm{H}, \mathrm{NH}) ; 3.96-3.94\left(\mathrm{~d}, 2 \mathrm{H}, \mathrm{CH}_{2}, \mathrm{~J}=4.8 \mathrm{~Hz}\right) ; 1.54$ (s, 9H, butyl- $\left.t\right) .{ }^{13} \mathrm{C}$ NMR: $172.7(\mathrm{COOH}) ; 157.4(\mathrm{C}=\mathrm{O}$, boc $) ; 79.6(\mathrm{C}-\alpha$, butyl- $t) ; 44.2(\mathrm{C}-\alpha$, gly $) ; 25.3$ (3C, C- $\beta$, butyl- $t$ ).

Anal. Calcd. for $\mathrm{C}_{7} \mathrm{H}_{13} \mathrm{NO}_{4}$ : C, 47.99; H, 7.48; N, 8.00. Found: C, 50.03; H, 7.46; N, 7.99.

tert-Butyloxycarbonyl-phenylalanine (2).

This compound was obtained according to the general procedure as white crystals, yield $88 \%, \mathrm{mp} 85-86^{\circ} \mathrm{C},[\alpha]_{\mathrm{D}}-4.0^{\circ}, \mathrm{R}_{\mathrm{f}}-0.78$; IR: $3298-2485(\mathrm{~m} / \mathrm{br}$, OH str, $\mathrm{COOH}$ ); 3089,3034 (w, CH str, ring); 2928 (m, CH str, asym, $\mathrm{CH}_{2}$ ); 1715 (s, C=O str, $\mathrm{COOH}$ ); 1632 (s, $\mathrm{C}=\mathrm{O}$ str, amide); 1582, 1482 (m, skeletal bands, ring); 1542 (m, NH bend, amide); 1387,1365 (m, CH bend, butyl- $t$ ); 933 (w, $\mathrm{CH}_{3}$ rock, butyl-t); 730, 693 (s, $\mathrm{CH}$ bend, oop, ring). ${ }^{1} \mathrm{H}$ NMR: 10.4 (br. $\mathrm{s}, 1 \mathrm{H}, \mathrm{OH}) ; 7.30-7.26(\mathrm{tt}, 2 \mathrm{H}, \mathrm{H}-\mathrm{m}) ; 7.10-7.08(\mathrm{dd}, 2 \mathrm{H}, \mathrm{H}-o, \mathrm{~J}=6.4 \mathrm{~Hz}, \mathrm{~J}=4.2$ Hz); 7.00-6.96 (t, 1H, H-p ) 5.89 (br. s, $1 \mathrm{H}, \mathrm{NH}$ ); 5.13-5.08 (q, 1H, H- $\alpha$ ); 3.25$3.23(\mathrm{~d}, 2 \mathrm{H}, \mathrm{J}=5.8 \mathrm{~Hz}, \mathrm{H}-\beta) ; 1.55$ (s, 9H, butyl-t). ${ }^{13} \mathrm{C}$ NMR: $173.8(\mathrm{COOH})$; $155.2(\mathrm{C}=\mathrm{O}$, boc $) ; 138.0(\mathrm{C}-\gamma) ; 129.3(2 \mathrm{C}, \mathrm{C}-o) ; 128.2(2 \mathrm{C}, \mathrm{C}-m) ; 126.5(\mathrm{C}-p)$; 79.5 (C- $\alpha$, butyl- $t) ; 55.1$ (C- $\alpha$ ); 37.7 (C- $\beta$ ); 27.8 (3C, C- $\beta$, butyl- $t$ ).

Anal. Calcd. for $\mathrm{C}_{14} \mathrm{H}_{19} \mathrm{NO}_{4}$ : C, 63.38; H, 7.22; N, 5.28. Found: C, 63.35; $\mathrm{H}, 7.23 ; \mathrm{N}, 5.32$.

General procedure for synthesis of L-amino acid methyl ester hydrochlorides (3-6)

Thionyl chloride $(1.4 \mathrm{~mL}, 20 \mathrm{mmol})$ was added to methanol $(100 \mathrm{~mL})$ slowly at $0{ }^{\circ} \mathrm{C}$ and L-amino acid $(20 \mathrm{mmol})$ was added to above solution. The resulting mixture was refluxed for $8-10 \mathrm{~h}$ at ambient temperature. Solvent was evaporated and the residue was triturated with ether at $0{ }^{\circ} \mathrm{C}$ until excess dimethyl sulphite was removed. The crude product was crystallized from methanol and 
ether at $0{ }^{\circ} \mathrm{C}$ to get pure amino acid methyl ester hydrochloride.

\section{L-Leucine methyl ester hydrochloride (3).}

This compound was obtained according to the general procedure as white crystals, yield $79 \%$, mp $118^{\circ} \mathrm{C},[\alpha]_{\mathrm{D}}-13.5^{\circ}, \mathrm{R}_{\mathrm{f}}-0.54$; IR: $3015-2852(\mathrm{~s} / \mathrm{br}$, $\mathrm{NH}_{3}{ }^{+}$str, asym and sym); 2925 (m, CH str, asym, $\left.\mathrm{CH}_{2}\right) ; 2822$ (m, CH str, $\mathrm{OCH}_{3}$ ); 1742 (s, $\mathrm{C}=\mathrm{O}$ str, ester); 1602, 1506 (s/br, $\mathrm{NH}_{3}^{+}$bend, asym and sym); 1390, 1369 (m, CH def, butyl-t); 1378, 1362 (s, CH def, propyl-i); 1269 (s, C-O str, ester); 931, 920 (w, $\mathrm{CH}_{3}$ rock, butyl- $t$ and propyl- $i$ ). ${ }^{1} \mathrm{H}$ NMR: 4.12 $\left(\mathrm{s}, 3 \mathrm{H}, \mathrm{OCH}_{3}\right)$; 4.03-3.99 (t, 2H, H- $\beta$ ); 3.55-3.52 (m, 1H, H- $\left.\alpha\right)$; 1.85-1.73 (m, $1 \mathrm{H}, \mathrm{H}-\gamma) ; 1.20-1.18$ (d, $6 \mathrm{H}, \mathrm{H}-\delta, \mathrm{J}=6.15 \mathrm{~Hz}) ; 1.17$ (br. s, $\mathrm{NH}_{3}^{+}$). ${ }^{13} \mathrm{C} \mathrm{NMR}$ : $169.5\left(\mathrm{C}=\mathrm{O}\right.$, ester); $53.0\left(\mathrm{OCH}_{3}\right) ; 49.6(\mathrm{C}-\alpha) ; 39.1(\mathrm{C}-\beta) ; 27.3(\mathrm{C}-\gamma) ; 20.9(2 \mathrm{C}$, $\mathrm{C}-\delta$ ).

Anal. Calcd. for $\mathrm{C}_{7} \mathrm{H}_{16} \mathrm{ClNO}_{2}: \mathrm{C}, 46.28 ; \mathrm{H}, 8.88 ; \mathrm{N}, 7.71$. Found: $\mathrm{C}, 46.25$; $\mathrm{H}, 8.90 ; \mathrm{N}, 7.69$.

\section{L-Tyrosine methyl ester hydrochloride (4).}

This compound was obtained according to the general procedure as white crystals, yield $84 \%, \mathrm{mp} 190^{\circ} \mathrm{C},[\alpha]_{\mathrm{D}}-4.3^{\circ}, \mathrm{R}_{\mathrm{f}}-0.67$; IR: 3372 (m/br, OH str); 3011-2863 (s/br, $\mathrm{NH}_{3}^{+}$str, asym and sym); 2928, 2848 (m, CH str, asym and sym, $\mathrm{CH}_{2}$ ); 1750 (s, C=O str, ester); 1588, 1475 (m, skeletal bands, ring); 1388, 1372 (m, CH def, butyl- $t$ ); 1227 (s, C-O str, phenolic); 1272 (s, C-O str, ester); 825 (s, CH def, oop, ring). ${ }^{1} \mathrm{H}$ NMR: 7.80-7.78 (dd, $2 \mathrm{H}, \mathrm{H}-\mathrm{m}, \mathrm{J}=7.4 \mathrm{~Hz}, \mathrm{~J}=5.0$ $\mathrm{Hz}$ ); 7.56-7.54 (dd, $2 \mathrm{H}, \mathrm{H}-\mathrm{o}, \mathrm{J}=7.5 \mathrm{~Hz}, \mathrm{~J}=4.6 \mathrm{~Hz}$ ), 5.40 (br. s, $\mathrm{OH}$ and $\mathrm{NH}_{3}^{+}$), 4.13-4.09 (m, $1 \mathrm{H}, \mathrm{H}-\alpha) ; 4.12\left(\mathrm{~s}, 3 \mathrm{H}, \mathrm{OCH}_{3}\right) ; 2.20-2.18(\mathrm{~d}, 2 \mathrm{H}, \mathrm{H}-\beta, \mathrm{J}=7.15$ Hz). ${ }^{13} \mathrm{C}$ NMR: $168.8(\mathrm{C}=\mathrm{O}$, ester); $156.5(\mathrm{C}-p) ; 130.2$ (2C, C-o ); $122.2(\mathrm{C}-\gamma)$; $117.5(2 \mathrm{C}, \mathrm{C}-m) ; 53.1\left(\mathrm{OCH}_{3}\right) ; 50.5(\mathrm{C}-\alpha) ; 32.9(\mathrm{C}-\beta)$.

Anal. Calcd. for $\mathrm{C}_{10} \mathrm{H}_{14} \mathrm{ClNO}_{3}: \mathrm{C}, 51.84 ; \mathrm{H}, 6.09 ; \mathrm{N}, 6.05$. Found: $\mathrm{C}, 51.82$; $\mathrm{H}, 6.10 ; \mathrm{N}, 6.06$.

\section{Glycine methyl ester hydrochloride (5).}

This compound was obtained according to the general procedure as white solid, yield $90 \%$, mp $174-175^{\circ} \mathrm{C},[\alpha]_{\mathrm{D}}-88.2^{\circ}, \mathrm{R}_{\mathrm{f}}-0.45$; IR: $3015-2853(\mathrm{~s} / \mathrm{br}$, $\mathrm{NH}_{3}{ }^{+}$str, asym and sym); 2925, 2847 (m, CH str, asym and sym, $\left.\mathrm{CH}_{2}\right) ; 1744$ (s, $\mathrm{C}=\mathrm{O}$ str, ester); 1599,1502 (s/br, $\mathrm{NH}_{3}^{+}$bend, asym and sym); 1386,1370 (m, CH def, butyl- $t$ ); 1268 (s, C-O str, ester), 932 (w, $\mathrm{CH}_{3}$ rock, butyl- $t$ ). ${ }^{1} \mathrm{H}$ NMR: 4.75 (br. s, $\left.\mathrm{NH}_{3}^{+}\right) ; 4.19$ (s, $\left.3 \mathrm{H}, \mathrm{OCH}_{3}\right) ; 3.85$ (m, $\left.2 \mathrm{H}, \mathrm{H}-\alpha\right) ;{ }^{13} \mathrm{C}$ NMR: $170.2\left(\mathrm{C}=\mathrm{O}\right.$, ester); $52.3\left(\mathrm{OCH}_{3}\right) ; 40.8(\mathrm{C}-\alpha)$.

Anal. Calcd. for $\mathrm{C}_{3} \mathrm{H}_{8} \mathrm{ClNO}_{2}$ : C, 28.70; H, 6.42; N, 11.16. Found: C, 28.69; $\mathrm{H}, 6.42 ; \mathrm{N}, 11.20$.

\section{L-Proline methyl ester hydrochloride (6).}

This compound was obtained according to the general procedure as viscous liquid, yield $87 \%,[\alpha]_{\mathrm{D}}-40.2^{\circ}, \mathrm{R}_{\mathrm{f}}-0.26$; IR: $3050-2840\left(\mathrm{~s} / \mathrm{br}, \mathrm{NH}_{2}^{+} \mathrm{str}\right.$, asym and sym); 2995, 2987 (m, CH str, $\mathrm{CH}_{2}$, pro); 1745 (s, C=O str, ester); 1440 (s/br, $\mathrm{NH}_{2}{ }^{+}$bend); 1270 (s, C-O str, ester). ${ }^{1} \mathrm{H}$ NMR: 4.73-4.70 (m, $\left.1 \mathrm{H}, \mathrm{H}-\alpha\right)$; $4.05-4.01^{2}(\mathrm{t}, 2 \mathrm{H}, \mathrm{H}-\delta) ; 3.86$ (s, 3H, $\left.\mathrm{OCH}_{3}\right) ; 2.45-2.32$ (m, 4H, H- $\left.\beta, \mathrm{H}-\gamma\right) ; 2.35$ (br. s, $\left.\mathrm{NH}_{2}^{+}\right) \cdot{ }^{13} \mathrm{C}$ NMR: $171.8(\mathrm{C}=\mathrm{O}$, ester $) ; 62.1(\mathrm{C}-\alpha) ; 54.5\left(\mathrm{OCH}_{3}\right) ; 44.7(\mathrm{C}-$ $\delta) ; 30.4(\mathrm{C}-\beta) ; 20.2(\mathrm{C}-\gamma)$.

Anal. Calcd. for $\mathrm{C}_{6} \mathrm{H}_{12} \mathrm{ClNO}_{2}: \mathrm{C}, 43.51 ; \mathrm{H}, 7.30 ; \mathrm{N}, 8.46$. Found: C, 43.49; $\mathrm{H}, 7.33 ; \mathrm{N}, 8.45$.

\section{1)}

General procedure for the synthesis of linear peptide fragments (7-

Peptide units were prepared according to Bodanzsky method with certain modifications ${ }^{29}$. Amino acid methyl ester hydrochloride/peptide methyl ester $(10 \mathrm{mmol})$ was dissolved in $\mathrm{CHCl}_{3}(20 \mathrm{~mL})$. To this, NMM $(21 \mathrm{mmol})$ was added at $0{ }^{\circ} \mathrm{C}$ and the reaction mixture was stirred for 15 minutes. Boc-amino acid/peptide $(10 \mathrm{mmol})$ in $\mathrm{CHCl}_{3}(20 \mathrm{~mL})$ and DCC $(10 \mathrm{mmol})$ were added with stirring. After $24 \mathrm{~h}$, the reaction mixture was filtered and the residue was washed with $\mathrm{CHCl}_{3}(30 \mathrm{~mL})$ and added to the filtrate. The filtrate was washed with $5 \% \mathrm{NaHCO}_{3}$ and saturated $\mathrm{NaCl}$ solutions. The organic layer was dried over anhydrous $\mathrm{Na}_{2} \mathrm{SO}_{4}$, filtered and evaporated in vacuum. The crude product was crystallized from a mixture of chloroform and petroleum ether followed by cooling at $0{ }^{\circ} \mathrm{C}$.

Deprotection at carboxyl terminal was done by adding lithium hydroxide $(0.36 \mathrm{~g}, 15 \mathrm{mmol})$ to a solution of Boc-di/tripeptide methyl ester $(10 \mathrm{mmol})$ in THF : $\mathrm{H}_{2} \mathrm{O}(1: 1,36 \mathrm{~mL})$ at $0{ }^{\circ} \mathrm{C}$. Resulting mixture was stirred at $\mathrm{RT}$ for $1 \mathrm{~h}$ and then acidified to $\mathrm{pH} 3.5$ with $1 \mathrm{~N} \mathrm{H}_{2} \mathrm{SO}_{4}$. The aqueous layer was extracted with
$\mathrm{Et}_{2} \mathrm{O}(3 \times 25 \mathrm{~mL})$. The combined organic extracts were dried over anhydrous $\mathrm{Na}_{2} \mathrm{SO}_{4}$ and concentrated under reduced pressure. The crude product was crystallized from methanol and ether to get pure Boc-di/tripeptides.

Deprotection at amino terminal was accomplished by treatment of Bocdi/tripeptides $(10 \mathrm{mmol})$ dissolved in $\mathrm{CHCl}_{3}(15 \mathrm{~mL})$ with trifluoroacetic acid $(2.28 \mathrm{~g}, 20 \mathrm{mmol})$. The resulting solution was stirred at RT for $1 \mathrm{~h}$, washed with saturated $\mathrm{NaHCO}_{3}$ solution $(25 \mathrm{~mL})$. The organic layer was dried over anhydrous $\mathrm{Na}_{2} \mathrm{SO}_{4}$ and concentrated under reduced pressure. The crude product was purified by crystallization from $\mathrm{CHCl}_{3}$ and petroleum ether (bp $40-60{ }^{\circ} \mathrm{C}$ ) to get pure di/tripeptide methyl esters.

\section{tert-Butyloxycarbonyl-glycyl-leucine methyl ester (7).}

This compound was obtained according to the general procedure as semisolid mass, yield $79 \%,[\alpha]_{\mathrm{D}}+1.8^{\circ}, \mathrm{R}_{\mathrm{f}}-0.36$; IR: 3125 (m, -NH str, amide); 2927 (m, - CH str, asym, $\left.\mathrm{CH}_{2}\right) ; 2853$ (m, - $\mathrm{CH}$ str, sym, $\left.\mathrm{CH}_{2}\right) ; 1742$ (s, $-\mathrm{C}=\mathrm{O}$ str, ester); 1642,1639 (s, $\mathrm{C}=\mathrm{O}$ str, $2^{\circ}$ amide); 1535,1529 (m, $-\mathrm{NH}$ bend, $2^{\circ}$ amide); 1392, 1368 (m, CH bend, butyl- $t$ ); 1380, 1363 (s, CH def, propyl- $i$ ); 1270 (s, $\mathrm{C}-\mathrm{O}$ str, ester); 930, 924 (w, $\mathrm{CH}_{3}$ rock, butyl- $t$ and propyl- $i$ ). ${ }^{1} \mathrm{H}$ NMR: 6.52 (br. s, $1 \mathrm{H}, \mathrm{NH}$ ); 6.02 (br. s, $1 \mathrm{H}, \mathrm{NH}$ ); 4.25-4.20 (q, $1 \mathrm{H}, \mathrm{H}-\alpha$, leu); 3.62 (s, $3 \mathrm{H}$, $\left.\mathrm{OCH}_{3}\right) ; 3.50-3.48\left(\mathrm{~d}, 2 \mathrm{H}, \mathrm{CH}_{2}\right.$, gly, J=4.75 Hz); 1.54 (s, 9H, butyl- $t$ ); 1.51-1.41 (m, 3H, H- $\beta, \mathrm{H}-\gamma$, leu); 0.96-0.94 (d, 6H, H- $\delta$, leu, J=6.1 Hz). ${ }^{13} \mathrm{C}$ NMR: 179.4 $(\mathrm{C}=\mathrm{O}$, ester $) ; 174.6(\mathrm{C}=\mathrm{O}$, amide); $155.8(\mathrm{C}=\mathrm{O}$, boc $) ; 79.4(\mathrm{C}-\alpha$, butyl- $t) ; 52.5$ $\left(\mathrm{OCH}_{3}\right) ; 49.2(\mathrm{C}-\alpha$, leu $) ; 46.8$ (C- $\alpha$, gly); 43.8 (C- $\beta$, leu); 29.3 (3C, C- $\beta$, butyl$t) ; 25.0(\mathrm{C}-\gamma$, leu $) ; 22.6(2 \mathrm{C}, \mathrm{C}-\delta$, leu $)$.

Anal. Calcd. for $\mathrm{C}_{14} \mathrm{H}_{26} \mathrm{~N}_{2} \mathrm{O}_{5}$ : C, 55.61; H, 8.67; N, 9.26. Found: C, 55.62; $\mathrm{H}, 8.65 ; \mathrm{N}, 9.25$.

tert-Butyloxycarbonyl-phenylalanyl-tyrosine methyl ester (8).

This compound was obtained according to the general procedure as white crystals, yield $77 \%$, mp $76^{\circ} \mathrm{C},[\alpha]_{\mathrm{D}}-9.6^{\circ}, \mathrm{R}_{\mathrm{f}}-0.74$; IR: 3373 (m/br, OH str, tyr); 3125 (m, NH str, amide); 3065, 3052 (w, CH str, rings); 2925, 2846 (m, $\mathrm{CH}$ str, asym and sym, $\mathrm{CH}_{2}$ ); 1749 (s, C=O str, ester); 1645,1638 (s, C=O str, amide); 1587, 1479, 1476 (m, skeletal bands, rings); 1536, 1526 (m, NH bend, amide); 1390, 1365 (m, CH bend, butyl-t); 1272 (s, C-O str, ester); 825, 710, 695 (s, CH def, oop, rings). ${ }^{1} \mathrm{H}$ NMR: 7.51-7.47 (tt, 2H, H-m, phe); 6.92-6.88 $(\mathrm{m}, 3 \mathrm{H}, \mathrm{H}-p$, phe and $\mathrm{H}-o$, tyr), 6.86-6.84 (dd, $2 \mathrm{H}, \mathrm{H}-o$, phe, $\mathrm{J}=6.45 \mathrm{~Hz}, \mathrm{~J}=4.15$ $\mathrm{Hz}$ ); 6.80-6.78 (dd, 2H, H-m, tyr, J=7.45 Hz, J=5.1 Hz); 6.66 (br. s, $1 \mathrm{H}, \mathrm{NH}$ ); 6.58 (br. s, 1H, NH); 5.95 (s, 1H, OH, tyr); 4.72-4.69 (q, 1H, H- $\alpha$, phe); 4.60$4.56\left(\mathrm{~m}, 1 \mathrm{H}, \mathrm{H}-\alpha\right.$, tyr); $3.54\left(\mathrm{~s}, 3 \mathrm{H}, \mathrm{OCH}_{3}\right) ; 3.02-2.76(\mathrm{~m}, 4 \mathrm{H}, \mathrm{H}-\beta$, phe and tyr); 1.55 (s, 9H, butyl- $t) .{ }^{13} \mathrm{C}$ NMR: $172.3(\mathrm{C}=\mathrm{O}$, phe); $169.6(\mathrm{C}=\mathrm{O}$, ester $)$; 153.8 (C- $p$, tyr); $152.0(\mathrm{C}=\mathrm{O}$, boc $) ; 133.3(\mathrm{C}-\gamma$, phe $) ; 130.8$ (2C, C-m, phe); 129.8 (2C, C-o, tyr); 129.1 (2C, C-o, phe); 128.9 (C-p, phe); 127.8 (C- $\gamma$, tyr); 126.0 (2C, C- $m$, tyr); 79.8 (C- $\alpha$, butyl- $t$ ); 57.3 (C- $\alpha$, phe); 49.3 (C- $\alpha$, tyr); 53.9 $\left(\mathrm{OCH}_{3}\right) ; 38.5,37.5$ (2C, C- $\beta$, phe and tyr); 27.5 (3C, C- $\beta$, butyl- $\left.t\right)$.

Anal. Calcd. for $\mathrm{C}_{24} \mathrm{H}_{30} \mathrm{~N}_{2} \mathrm{O}_{6}: \mathrm{C}, 65.14 ; \mathrm{H}, 6.83 ; \mathrm{N}, 6.33$. Found: C, 65.15; $\mathrm{H}, 6.85 ; \mathrm{N}, 6.32$.

\section{tert-Butyloxycarbonyl-glycyl-leucyl-glycine methyl ester (9).}

This compound was obtained according to the general procedure as semisolid mass, yield $79 \%,[\alpha]_{\mathrm{D}}+19.6^{\circ}, \mathrm{R}_{\mathrm{f}}-0.28$; IR: $3126(\mathrm{~m}, \mathrm{NH}$ str, amide); 2924, 2850, 2845 (m, CH str, asym and sym, $\mathrm{CH}_{2}$ ); 1752 (s, C=O str, ester); 1644, 1638 (s, C=O str, amide); 1532, 1525 (m, NH bend, amide); 1389, 1366 (m, CH bend, butyl- $t$ ); 1382, 1360 (s, CH def, propyl- $i$ ); 1268 (s, C-O str, ester); 932, 920 (w, $\mathrm{CH}_{3}$ rock, butyl- $t$ and propyl- $i$ ). ${ }^{1} \mathrm{H}$ NMR: 7.56, 7.15, 7.10 (br. s, $3 \mathrm{H}, \mathrm{NH})$; 4.57-4.53 (q, $1 \mathrm{H}, \mathrm{H}-\alpha$, leu); 4.03-4.01 (d, $2 \mathrm{H}, \mathrm{CH}_{2}$, gly-2, $\mathrm{J}=4.75$ $\mathrm{Hz})$; 3.74-3.72 (d, $2 \mathrm{H}, \mathrm{CH}_{2}$, gly-1, J=4.8 Hz); 3.65 (s, 3H, $\left.\mathrm{OCH}_{3}\right) ; 1.86-1.82$ (t, $2 \mathrm{H}, \mathrm{H}-\beta, \mathrm{leu}) ; 1.54$ (s, 9H, butyl- $t$ ); 1.50-1.42 (m, $1 \mathrm{H}, \mathrm{H}-\gamma, \mathrm{leu}) ; 0.99-0.97$ (d, $6 \mathrm{H}, \mathrm{H}-\delta$, leu, $\mathrm{J}=6.1 \mathrm{~Hz}) \cdot{ }^{13} \mathrm{C}$ NMR: $171.2(\mathrm{C}=\mathrm{O}$, leu $) ; 169.3(\mathrm{C}=\mathrm{O}$, ester $) ;$ $166.1\left(\mathrm{C}=\mathrm{O}\right.$, gly-1); $158.0(\mathrm{C}=\mathrm{O}$, boc $) ; 79.4(\mathrm{C}-\alpha$, butyl- $t) ; 53.2\left(\mathrm{OCH}_{3}\right) ; 47.8$ (C- $\alpha$, leu); 44.1 (C- $\alpha$, gly-1), 40.1 (C- $\beta$, leu); 41.5 (C- $\alpha$, gly-2), 30.2 (3C, C- $\beta$, butyl-t); 25.9 (C- $\gamma$, leu); 22.8 (2C, C- $\delta$, leu).

Anal. Calcd. for $\mathrm{C}_{16} \mathrm{H}_{29} \mathrm{~N}_{3} \mathrm{O}_{6}: \mathrm{C}, 53.47 ; \mathrm{H}, 8.13 ; \mathrm{N}, 11.69$. Found: C, 53.45; $\mathrm{H}, 8.15 ; \mathrm{N}, 11.70$. (10).

tert-Butyloxycarbonyl-phenylalanyl-tyrosinyl-proline methyl ester

This compound was obtained according to the general procedure as semisolid mass, yield $76 \%,[\alpha]_{\mathrm{D}}-65.0^{\circ}, \mathrm{R}_{\mathrm{f}}-0.82 ; \mathrm{IR}: 3369(\mathrm{~m} / \mathrm{br}$, OH str, tyr); 3128, 3122 (m, NH str, amide); 3066, 3041 (w, CH str, rings); 2996, 2982 (m, $\mathrm{CH}$ str, $\mathrm{CH}_{2}$, pro); 2928, 2842 (m, CH str, asym and sym, $\left.\mathrm{CH}_{2}\right) ; 1753$ (s, C=O 
str, ester); $1669,1640,1636$ (s, $\mathrm{C}=\mathrm{O}$ str, $3^{\circ}$ and $2^{\circ}$ amide); 1589, 1581, 1478 , 1473 (m, skeletal bands, rings); 1535, 1522 (m, NH bend, amide); 1392, 1366 (m, CH bend, butyl- $t$ ); 1270 (s, C-O str, ester); 822, 713, 696 (s, CH def, oop, rings). ${ }^{1} \mathrm{H}$ NMR: 8.65 (br. s, $\left.1 \mathrm{H}, \mathrm{NH}\right) ; 7.51-7.48$ (tt, $2 \mathrm{H}, \mathrm{H}-m$, phe); 6.99-6.97 (dd, $2 \mathrm{H}, \mathrm{H}-m$, tyr, J=7.5 Hz, J=5.15 Hz); 6.92-6.89 (m, 3H, H-p, phe and $\mathrm{H}-o$, tyr), 6.85-6.83 (dd, 2H, H-o, phe, J=6.5 Hz, J=4.1 Hz); 6.56 (br. s, 1H, NH); $5.94(\mathrm{~s}, 1 \mathrm{H}, \mathrm{OH}, \mathrm{tyr}) ; 4.61-4.52(\mathrm{~m}, 2 \mathrm{H}, \mathrm{H}-\alpha$, phe and tyr); 3.94-3.89 (t, $1 \mathrm{H}$, $\mathrm{H}-\alpha$, pro); 3.62 (s, $\left.3 \mathrm{H}, \mathrm{OCH}_{3}\right) ; 3.40-3.36$ (t, $2 \mathrm{H}, \mathrm{H}-\delta$, pro); 2.97-2.82 (m, $4 \mathrm{H}$, $\mathrm{H}-\beta$, phe and tyr); $2.06-1.96$ (m, $4 \mathrm{H}, \mathrm{H}-\beta, \mathrm{H}-\gamma$, pro); 1.55 (s, $9 \mathrm{H}$, butyl $-t) .{ }^{13} \mathrm{C}$ NMR: $176.1(\mathrm{C}=\mathrm{O}$, phe $) ; 169.2(\mathrm{C}=\mathrm{O}$, ester $) ; 167.8(\mathrm{C}=\mathrm{O}$, tyr $) ; 153.9(\mathrm{C}=\mathrm{O}$, boc); 153.4 (C- $p$, tyr); 133.5 (C- $\gamma$, phe); 130.9 (2C, C-m, phe); 129.7, 129.2 (4C, C- $o$, tyr and phe); 128.9 (C- $\gamma$, tyr); 128.4 (C- $p$, phe); 125.2 (2C, C- $m$, tyr); $79.9(\mathrm{C}-\alpha$, butyl- $t) ; 58.2(\mathrm{C}-\alpha$, pro $) ; 54.1\left(\mathrm{OCH}_{3}\right) ; 51.2,48.8(2 \mathrm{C}, \mathrm{C}-\alpha$, phe and tyr); 45.2 (C- $\delta$, pro); 38.0, 37.6 (2C, C- $\beta$, phe and tyr); 28.7 (C- $\beta$, pro); 27.3 (3C, C- $\beta$, butyl- $t$ ); 24.3 (C- $\gamma$, pro).

Anal. Calcd. for $\mathrm{C}_{29} \mathrm{H}_{37} \mathrm{~N}_{3} \mathrm{O}_{7}$ : C, 64.55; H, 6.91; N, 7.79. Found: C, 64.55; $\mathrm{H}, 6.90 ; \mathrm{N}, 7.82$.

tert-Butyloxycarbonyl-glycyl-leucyl-glycyl-phenylalanyl-tyrosinylproline methyl ester (11).

This compound was obtained according to the general procedure as semisolid mass, yield $88 \%,[\alpha]_{\mathrm{D}}-11.2^{\circ}, \mathrm{R}_{\mathrm{f}}-0.43$; IR: 3365 (m/br, OH str, tyr); 3132, 3129, 3123 (m, NH str, amide); 3062 (w, CH str, rings); 2995, 2980 (m, $\mathrm{CH}$ str, $\mathrm{CH}_{2}$, pro); 2929, 2852-2849 (m, CH str, asym and sym, $\left.\mathrm{CH}_{2}\right) ; 1750$ (s, $\mathrm{C}=\mathrm{O}$ str, ester); $1672,1639,1636\left(\mathrm{~s}, \mathrm{C}=\mathrm{O}\right.$ str, $3^{\circ}$ and $2^{\circ}$ amide); 1586,1580 , 1476, 1469 (m, skeletal bands, rings); 1535, 1532-1524 (m, NH bend, amide); 1390,1365 (m, CH bend, butyl- $t$ ); 1382, 1359 (s, CH def, propyl- $i$ ); 1272 (s, C-O str, ester); 929, 922 (w, $\mathrm{CH}_{3}$ rock, butyl- $t$ and propyl-i); 824, 712, 696 (s, $\mathrm{CH}$ def, oop, rings); 1.55 (s, 9H, butyl- $t$ ). ${ }^{1} \mathrm{H}$ NMR: 9.49, 8.68, 8.02, 7.51 (br. s, 4H, NH); 7.21-7.16 (tt, 2H, H-m, phe); 7.05-7.02 (t, 1H, H-p, phe), 6.98-6.88 (m, 4H, H-o, H- $m$, tyr); 6.83-6.81 (dd, 2H, H-o, phe, J=6.5 Hz, J=4.15 Hz); 7.52 (br. s, $1 \mathrm{H}, \mathrm{NH}) ; 5.98$ (s, $1 \mathrm{H}, \mathrm{OH}, \mathrm{tyr}) ; 4.41-4.34$ (q, $1 \mathrm{H}, \mathrm{H}-\alpha, \mathrm{tyr}) ; 4.26-$ 4.21 (q, $1 \mathrm{H}, \mathrm{H}-\alpha$, phe); 4.20-4.15 (q, $1 \mathrm{H}, \mathrm{H}-\alpha$, leu); 4.03-4.01 (d, $2 \mathrm{H}, \mathrm{CH}_{2}$, gly-2, J=4.75 Hz); 3.94-3.91 (t, $1 \mathrm{H}, \mathrm{H}-\alpha$, pro); 3.62 (s, 3H, OCH $) ; 3.53-3.51$ (d, $2 \mathrm{H}, \mathrm{CH}_{2}$, gly-1, J=4.8 Hz); 3.41-3.38 (t, $2 \mathrm{H}, \mathrm{H}-\delta$, pro); 2.81-2.72 (m, $4 \mathrm{H}$, $\mathrm{H}-\beta$, phe and tyr); $2.09-1.95$ (m, $4 \mathrm{H}, \mathrm{H}-\beta, \mathrm{H}-\gamma$, pro); $1.86-1.83$ (t, $2 \mathrm{H}, \mathrm{H}-\beta$ leu); 1.54 (s, 9H, butyl-t); 1.51-1.40 (m, 1H, H- $\gamma$, leu); 1.03-1.01 (d, $6 \mathrm{H}, \mathrm{H}-\delta$, leu, J=5.95 Hz). ${ }^{13} \mathrm{C}$ NMR: $175.3(\mathrm{C}=\mathrm{O}$, gly-2); $168.9(\mathrm{C}=\mathrm{O}, \mathrm{tyr}) ; 167.9(\mathrm{C}=\mathrm{O}$, ester); $165.7(\mathrm{C}=\mathrm{O}$, gly-1); $166.3(\mathrm{C}=\mathrm{O}$, leu $) ; 165.7(\mathrm{C}=\mathrm{O}$, phe $) ; 157.5(\mathrm{C}=\mathrm{O}$, boc); 153.9 (C- $p$, tyr); 139.4 (C- $\gamma$, phe); 135.6 (C- $\gamma$, tyr); 130.4, 129.1 (4C, C$o$, C- $m$, phe); $128.3,127.8$ (4C, C- $o$, C- $m$, tyr); 127.2 (C- $p$, phe); 79.5 (C- $\alpha$, butyl- $t) ; 57.3,55.5\left(2 \mathrm{C}, \mathrm{C}-\alpha\right.$, pro and tyr); $53.5\left(\mathrm{OCH}_{3}\right) ; 52.6,47.6(2 \mathrm{C}, \mathrm{C}-\alpha$, phe and leu); 46.0 (C- $\alpha$, gly-2); 45.3 (C- $\delta$, pro); 44.1 (C- $\alpha$, gly-1); 39.6 (C- $\beta$, leu); $37.2,36.8$ (2C, C- $\beta$, phe and tyr); 30.7 (C- $\beta$, pro); 29.2 (3C, C- $\beta$, butyl- $t$ ); 27.5 (C- $\gamma$, leu); 24.5 (C- $\gamma$, pro); 22.2 (2C, C- $\delta$, leu).

Anal. Calcd. for $\mathrm{C}_{39} \mathrm{H}_{54} \mathrm{~N}_{6} \mathrm{O}_{10}$ : C, 61.08; H, 7.10; N, 10.96. Found: C, $61.05 ; \mathrm{H}, 7.10 ; \mathrm{N}, 11.00$.

Procedure for synthesis of cyclohexapeptide, cherimolacyclopeptide E (12)

To synthesize compound 12, linear hexapeptide unit $11(5 \mathrm{mmol})$ was deprotected at carboxyl end using $\mathrm{LiOH}(0.18 \mathrm{~g}, 7.5 \mathrm{mmol})$ to get Boc-glyleu-gly-phe-tyr-pro-OH. The deprotected hexapeptide unit $(5 \mathrm{mmol})$ was now dissolved in $\mathrm{CHCl}_{3}(50 \mathrm{~mL})$ at $0{ }^{\circ} \mathrm{C}$. To the above solution, p-nitrophenol (pnp) $(0.94 \mathrm{~g}, 6.7 \mathrm{mmol})$ was added and stirred at RT for $12 \mathrm{~h}$. The reaction mixture was filtered and the filtrate was washed with $10 \% \mathrm{NaHCO}_{3}$ solution $(3 \times 15$ $\mathrm{mL}$ ) until excess of p-nitrophenol was removed and finally washed with $5 \%$ $\mathrm{HCl}(2 \times 10 \mathrm{~mL})$ to get the corresponding p-nitrophenyl ester Boc-gly-leugly-phe-tyr-pro-O-pnp. To this compound $(4 \mathrm{mmol})$ dissolved in $\mathrm{CHCl}_{3}$ (35 $\mathrm{mL}), \mathrm{CF}_{3} \mathrm{COOH}(0.91 \mathrm{~g}, 8 \mathrm{mmol})$ was added, stirred at RT for $1 \mathrm{~h}$ and washed with $10 \% \mathrm{NaHCO}_{3}$ solution $(2 \times 25 \mathrm{~mL})$. The organic layer was dried over anhydrous $\mathrm{Na}_{2} \mathrm{SO}_{3}$ to get Gly-leu-gly-phe-tyr-pro-O-pnp which was dissolved in $\mathrm{CHCl}_{3}\left(25^{2} \mathrm{~mL}\right)$ and TEA/NMM/C $\mathrm{H}_{5} \mathrm{~N}(2.8 \mathrm{~mL} / 2.21 \mathrm{~mL} / 1.61 \mathrm{~mL}, 20$ $\mathrm{mmol}$ ) was added. Then, whole contents were kept at $0{ }^{\circ} \mathrm{C}$ for 7 days. The reaction mixture was washed with $10 \% \mathrm{NaHCO}_{3}$ solution until the byproduct p-nitrophenol was removed completely and finally washed with $5 \% \mathrm{HCl}(3$ $\times 15 \mathrm{~mL}$ ). The organic layer was dried over anhydrous $\mathrm{Na}_{2} \mathrm{SO}_{4}$. Finally, chloroform was distilled off and crude cyclized product was crystallized from $\mathrm{CHCl}_{3}$ and n-hexane.
Cyclo (gly-leu-gly-phe-tyr-pro) (12).

This compound was obtained according to the above mentioned procedure as white crystals, yield $75 \%(\mathrm{NMM}), 61 \%(\mathrm{TEA}), 56 \%\left(\mathrm{C}_{5} \mathrm{H}_{5} \mathrm{~N}\right), \mathrm{mp} 213{ }^{\circ} \mathrm{C}$ $\left(213-214{ }^{\circ} \mathrm{C}\right),[\alpha]_{\mathrm{D}}-55.7^{\circ}\left(-56.0^{\circ}\right), \mathrm{R}_{\mathrm{f}}-0.65$; IR: $3372(\mathrm{~m} / \mathrm{br}, \mathrm{OH}$ str, tyr); 3129-3122 (m, NH str, amide); 3074, 3052 (w, CH str, rings); 2998-2993 (m, $\mathrm{CH}$ str, $\mathrm{CH}_{2}$, pro); 2926-2923, 2852-2844 (m, CH str, asym and sym, $\mathrm{CH}_{2}$ ); $1673,1645-1636\left(\mathrm{~s}, \mathrm{C}=\mathrm{O}\right.$ str, $3^{\circ}$ and $2^{\circ}$ amide); $1585,1582,1472(\mathrm{~m}$, skeletal bands, rings); 1536-1530, 1525-1518 (m, NH bend, amide); 1380, 1361 (s, $\mathrm{CH}$ def, propyl-i); 1233 (s, C-O str, tyr); 922 (w, $\mathrm{CH}_{3}$ rock, propyl-i); 825, 720, 688 (s, CH def, oop, rings). ${ }^{1} \mathrm{H}$ NMR: $9.18,8.10,7.61,7.54$ (br. s, $4 \mathrm{H}, \mathrm{NH}$ ); 7.20-7.16 (tt, 2H, H-m, phe); 7.10 (br. s, 1H, NH); 7.02-6.99 (t, 1H, H-p, phe), 6.98-6.89 (m, 4H, H-o, H- $m$, tyr); 6.84-6.82 (dd, $2 \mathrm{H}, \mathrm{H}-o$, phe, J=6.45 Hz, $\mathrm{J}=4.1 \mathrm{~Hz}) ; 6.26-6.22(\mathrm{q}, 1 \mathrm{H}, \mathrm{H}-\alpha, \mathrm{leu}) ; 5.96(\mathrm{~s}, 1 \mathrm{H}, \mathrm{OH}, \mathrm{tyr}) ; 5.75-5.71(\mathrm{~m}$, $1 \mathrm{H}, \mathrm{H}-\alpha$, tyr); 5.65-5.61 (q, $1 \mathrm{H}, \mathrm{H}-\alpha$, phe); 5.24-5.22 (d, $2 \mathrm{H}, \mathrm{CH}_{2}$, gly-2, J=4.8 $\mathrm{Hz}) ; 3.96-3.94$ (d, 2H, CH , gly-1, J=4.75 Hz); 3.90-3.87 (t, $1 \mathrm{H}, \mathrm{H}-\alpha$, pro); 3.28-3.24 (t, $2 \mathrm{H}, \mathrm{H}-\delta$, pro); $2.69-2.65$ (m, $2 \mathrm{H}, \mathrm{H}-\beta$, pro); $2.62-2.58(\mathrm{~m}, 4 \mathrm{H}$, $\mathrm{H}-\beta$, phe and tyr); $1.89-1.78(\mathrm{~m}, 4 \mathrm{H}, \mathrm{H}-\gamma$, pro and leu); 0.99-0.97 (d, $6 \mathrm{H}, \mathrm{H}-\delta$, leu, $\mathrm{J}=6.0 \mathrm{~Hz}) ; 0.86-0.75(\mathrm{~m}, 1 \mathrm{H}, \mathrm{H}-\gamma$, leu $) .{ }^{13} \mathrm{C}$ NMR: $172.9(\mathrm{C}=\mathrm{O}$, pro $) ; 171.5$, $170.6(\mathrm{C}=\mathrm{O}$, leu and phe $) ; 169.9(\mathrm{C}=\mathrm{O}$, tyr $) ; 168.5,167.3(\mathrm{C}=\mathrm{O}$, gly- 1 and gly2); 154.2 (C- $p$, tyr); 137.9 (C- $p$, phe); 132.8 (C- $\gamma$, tyr); 129.4, 128.5 (2C, C-o, C- $m$, phe); 126.7 (2C, C-o, tyr); 124.2 (C- $\gamma$, phe); 122.3 (2C, C- $m$, tyr); 62.2, 56.9 (2C, C- $\alpha$, pro and tyr); 52.3, 51.6 (2C, C- $\alpha$, leu and phe); 48.0 (C- $\delta$, pro); 43.9 (C- $\beta$, leu); 42.9 (C- $\alpha$, gly-2); 40.6 (C- $\alpha$, gly-1); 38.6, 37.5 (2C, C- $\beta$, phe and tyr); 30.9 (C- $\beta$, pro); 27.2 (C- $\gamma$, leu); 24.7 (C- $\gamma$, pro); 22.5 (2C, C- $\delta$, leu). MS (FAB): $m / z[\%]=635.7\left[\left(\mathrm{M}^{+}+1\right)^{+}, 100\right], 607.7\left[(635.7-\mathrm{CO})^{+}, 32\right], 488.5$ [(tyr-pro-gly-leu-gly $\left.)^{+}, 28\right], 460.5\left[(488.5-\mathrm{CO})^{+}, 12\right], 431.5$ [(tyr-pro-gly-leu $)^{+}$,

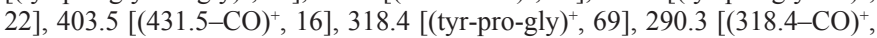

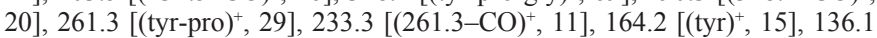
$\left[(164.2-\mathrm{CO})^{+}, 10\right], 107.1\left[\left(\mathrm{C}_{7} \mathrm{H}_{7} \mathrm{O}\right)^{+}, 6\right], 93.1\left[\left(\mathrm{C}_{6} \mathrm{H}_{5} \mathrm{O}\right)^{+}, 4\right], 91.0\left[\left(\mathrm{C}_{7} \mathrm{H}_{7}\right)^{+}, 5\right]$, $57.1\left[\left(\mathrm{C}_{4} \mathrm{H}_{9}\right)^{+}, 9\right], 43.1\left[\left(\mathrm{C}_{3} \mathrm{H}_{7}\right)^{+}, 9\right], 30.0\left[\left(\mathrm{CH}_{4} \mathrm{~N}\right)^{+}, 4\right], 29.0\left[\left(\mathrm{C}_{2} \mathrm{H}_{5}\right)^{+}, 4\right], 15.0$ $\left[\left(\mathrm{CH}_{3}\right)^{+}, 7\right]$

Anal. Calcd. for $\mathrm{C}_{33} \mathrm{H}_{42} \mathrm{~N}_{6} \mathrm{O}_{7}$ : C, 62.45; H, 6.67; N, 13.24. Found: C, 62.42; $\mathrm{H}, 6.70 ; \mathrm{N}, 13.25$.

\section{PHARMACOLOGICAL EVALUATION}

The newly synthesized cyclohexapeptide $\mathbf{1 2}$ was evaluated for in vitro antibacterial, antifungal, anthelmintic and cytotoxic activities. The antimicrobial activity was carried out against gram positive bacteria Bacillus subtilis (MUMC 408), Staphylococcus aureus (MUMC 377), gram negative bacteria Pseudomonas aeruginosa (MUMC 266), Escherichia coli (MUMC 106) and cutaneous fungi Microsporum audouinii (MUMC 545) and Trichophyton mentagrophytes (MUMC 665), diamorphic fungi Candida albicans (MUMC 29) and pathogenic fungi Aspergillus niger (MUMC 188) using ciprofloxacin and griseofulvin as reference drugs. Anthelmintic activity was performed against three earthworm species Megascoplex konkanensis (ICARBC 211), Pontoscotex corethruses (ICARBC 408) and Eudrilus sp. (ICARBC 042) using mebendazole and piperazine citrate as standard drugs. The cytotoxic activity was tested against Dalton's Lymphoma Ascites (DLA) cells and Ehrlich's Ascites Carcinoma (EAC) cells using 5-fluorouracil (5-FU) as reference compound. Bacterial and fungal cultures were obtained from the Manipal University Mycological Center (MUMC, Manipal, India) and earthworm species were obtained from Indian Council of Agricultural Research Breeding Center (ICARBC, Kasaragod, India).

\section{Antimicrobial screening}

Antimicrobial activity study was carried out against eight pathogenic microorganisms at 12.5-6 $\mu \mathrm{g} \mathrm{mL} \mathrm{m}^{-1}$ concentration using Kirby-Bauer disk diffusion method ${ }^{30}$. MIC values of test compound were determined by Tube Dilution Technique using DMF and DMSO. A spore suspension in sterile distilled water was prepared from 5 days old culture of the test bacteria/fungi growing on nutrient broth media/sabouraud's broth media. About $20 \mathrm{~mL}$ of the growth medium was transferred into sterilized petri plates and inoculated with $1.5 \mathrm{~mL}$ of the spore suspension (spore concentration $-6 \times 10^{4}$ spores $\mathrm{mL}^{-1}$ ). Filter paper disks of $6 \mathrm{~mm}$ diameter and $2 \mathrm{~mm}$ thickness were sterilized by autoclaving at $121{ }^{\circ} \mathrm{C}$ for $15 \mathrm{~min}$. Each petri plate was divided into five equal portions along the diameter to place one disc. Three discs of test sample 12 were placed on three portions together with one disc with reference drug ciprofloxacin/griseofulvin and a disk impregnated with the solvent (DMF/ DMSO) as negative control. Reference drugs were also tested at the same concentration of $12.5-6 \mu \mathrm{g} \mathrm{mL} L^{-1}$. The petri plates inoculated with bacterial/ fungal cultures were incubated at $37{ }^{\circ} \mathrm{C}$ for $18 \mathrm{~h}$ and $48 \mathrm{~h}$, respectively. Diameters of the zones of inhibition (in $\mathrm{mm}$ ) were measured and the average 
diameters for test sample were calculated for triplicate sets triplicate sets. The diameters obtained for the test sample $\mathbf{1 2}$ were compared with that produced by the standard drugs.

\section{Anthelmintic screening}

Anthelmintic activity study was carried out against three different species of earthworms at $2 \mathrm{mg} \mathrm{mL}^{-1}$ concentration using Garg method ${ }^{31}$. Suspension of sample was prepared by triturating synthesized compound $12(0.1 \mathrm{~g})$ with tween $80(0.5 \%)$ and distilled water and the resulting mixture was stirred using a mechanical stirrer for 30 minutes. The suspension was diluted to contain $0.2 \% \mathrm{w} / \mathrm{v}$ of the test sample. Suspension of reference drugs, mebendazole and piperazine citrate were prepared with the same concentration in a similar way. Three sets of five earthworms of almost similar sizes ( 2 inch in length) were placed in petri plates of 4 inch diameter containing $50 \mathrm{~mL}$ of suspension of test sample and reference drugs at RT. Another set of five earthworms was kept as control in $50 \mathrm{~mL}$ suspension of distilled water and tween $80(0.5 \%)$. The paralyzing and death times were noted and their mean was calculated for triplicate sets. The death time was ascertained by placing the earthworms in warm water $\left(50{ }^{\circ} \mathrm{C}\right)$ which stimulated the movement, if the worm was alive.

\section{Cytotoxicity screening}

Synthesized cyclopeptide 12 was subjected to short term in vitro cytotoxicity study at $62.5-3.91 \mu \mathrm{g} \mathrm{mL}^{-1}$ using Kuttan method ${ }^{32}$. Activity was assessed by determining the percentage inhibition of DLA and EAC cells. Both cells were cultured in the peritoneal cavity of healthy albino mice by injecting the suspension of cells $\left(1 \times 10^{6}\right.$ cells $\left./ \mathrm{mL}\right)$ intraperitoneally. After 15-20 days, cells were withdrawn from the peritoneal cavity of the mice with help of sterile syringe and counted using haemocytometer and adjusted to $1 \times 10^{6}$ cells $/ \mathrm{mL}$. Different dilution of test compound 12 ranging from $62.5-3.91 \mu \mathrm{g} \mathrm{mL}^{-1}$ were prepared in dulbecoccs minimum essential medium and $0.1 \mathrm{~mL}$ of each diluted test compound was added to $0.1 \mathrm{~mL}$ of DLA cells $\left(1 \times 10^{6}\right.$ cells $\left./ \mathrm{mL}\right)$ and EAC cells $\left(1 \times 10^{6}\right.$ cells $\left./ \mathrm{mL}\right)$. Resulting suspensions were incubated at $37^{\circ} \mathrm{C}$ for 3 $\mathrm{h}$. After $3 \mathrm{~h}$, tryphan blue dye exclusion test was performed and percentage growth inhibition was calculated. $\mathrm{CTC}_{50}$ values were determined by graphical extrapolation method. Controls were also tested at $62.5-3.91 \mu \mathrm{g} \mathrm{mL}^{-1}$ against both cell lines.

\section{RESULTS AND DISCUSSION}

\section{CHEMISTRY}

In present work, disconnection strategy was employed to carry out the first total synthesis of cherimolacyclopeptide E (12). The cyclic hexapeptide molecule was split into two amino acid units Gly- $\mathrm{OCH}_{3} \cdot \mathrm{HCl}(5), \mathrm{Pro}_{-} \mathrm{OCH}_{3}$. $\mathrm{HCl}(6)$ and two dipeptide units Boc-gly-leu-OCH $\mathrm{OCH}_{3}$ (7), Boc-phe-tyr-OCH $\mathbf{O C H}_{3}$. The required dipeptide units 7,8 were prepared by coupling of Boc-amino acids viz. Boc-gly (1) and Boc-phe (2) with corresponding amino acid methyl ester hydrochlorides such as Leu- $\mathrm{OCH}_{3} \cdot \mathrm{HCl}(3), \mathrm{Tyr}_{-} \mathrm{OCH}_{3} \cdot \mathrm{HCl}(4)$ employing dicyclohexylcarbodiimide (DCC) as coupling agent. Ester group of dipeptide 7 was removed by alkaline hydrolysis with $\mathrm{LiOH}$ to get Boc-gly-leu-OH which was coupled with amino acid methyl ester hydrochloride $\mathbf{5}$ using DCC and $\mathrm{N}$-methylmorpholine (NMM), to get the first tripeptide unit Boc-gly-leu-gly$\mathrm{OCH}_{3}(\mathbf{9})$, Similarly, dipeptide $\mathbf{8}$ was deprotected at carboxyl end to get Bocphe-tyr-OH and coupled with amino acid methyl ester hydrochloride 6 to get another tripeptide unit Boc-phe-tyr-pro- $\mathrm{OCH}_{3}$ (10). After removal of ester group of tripeptide 9 and Boc group of tripeptide 10, deprotected units were coupled with each other to get linear hexapeptide unit Boc-gly-leu-gly-phe-tyrpro- $\mathrm{OCH}_{3}(\mathbf{1 1})$. The ester group of linear fragment was removed using $\mathrm{LiOH}$ and p-nitrophenyl (pnp) ester group was introduced. After removing Bocgroup of resulting unit Boc-gly-leu-gly-phe-tyr-pro-Opnp with $\mathrm{CF}_{3} \mathrm{COOH}$, deprotected linear fragment was cyclized by keeping the whole contents at 0 ${ }^{\circ} \mathrm{C}$ for 7 days in presence of catalytic amount of TEA/NMM/pyridine to get cyclic compound 12 (Scheme 1).

Synthesis of cyclohexapeptide $\mathbf{1 2}$ and intermediate linear peptide segments 1-11 was carried out successfully with good yield $(>75 \%)$ and NMM was proved to be a yield effective base for cyclization of linear hexapeptide unit in comparison to TEA and pyridine. IR spectra of all peptide units 7-11 showed characteristic medium to strong bands corresponding to carbonyl stretching at

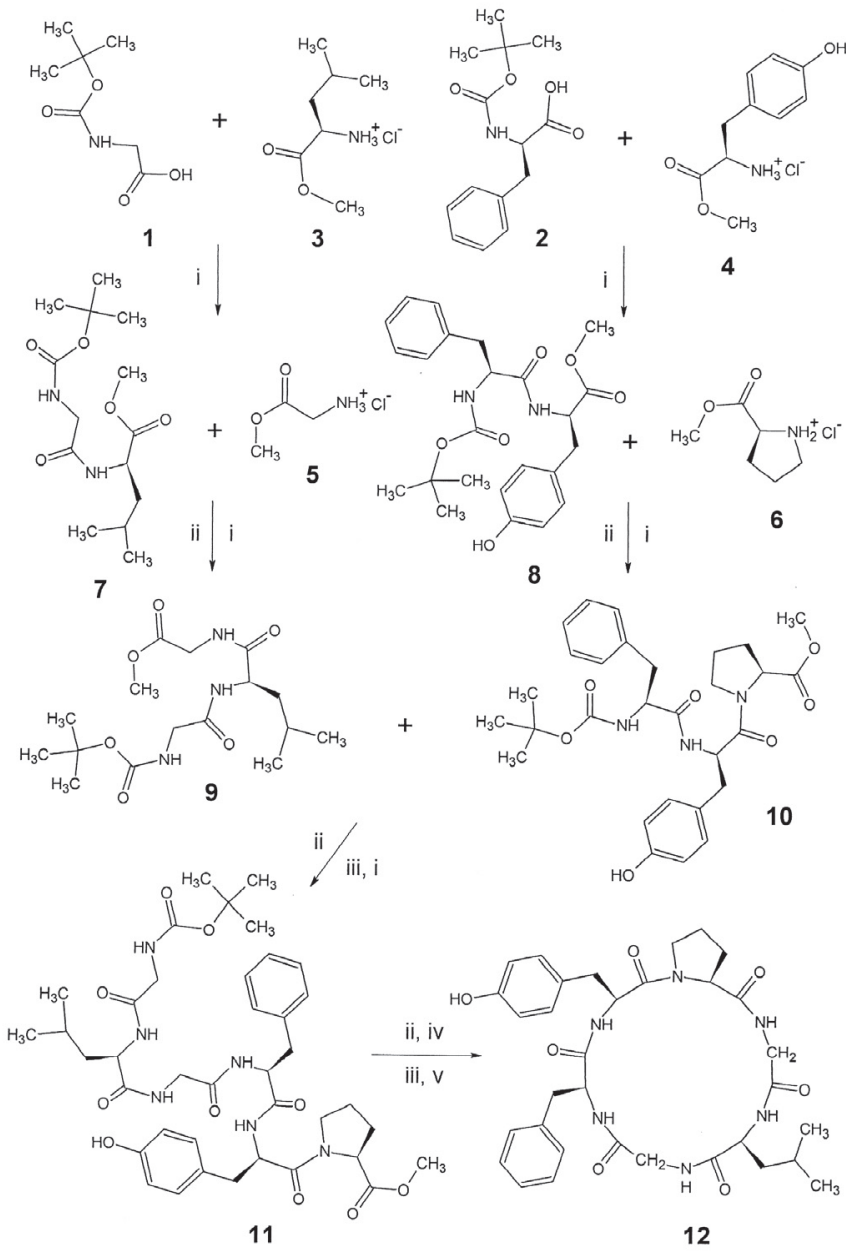

i) DCC, NMM, $\mathrm{CHCl}_{3}, \mathrm{RT}, 24 \mathrm{~h}$; ii) LiOH, THF: $\mathrm{H}_{2} \mathrm{O}(1: 1)$, RT, $1 \mathrm{~h}$; iii) TFA, $\mathrm{CHCl}_{3}, \mathrm{RT}, 1 \mathrm{~h}$; iv) pnp, $\mathrm{CHCl}_{3}, \mathrm{RT}, 12 \mathrm{~h}$; v) TEA/NMM/ $\mathrm{C}_{5} \mathrm{H}_{5} \mathrm{~N}, 7$ days, $0{ }^{\circ} \mathrm{C}$

Scheme 1. Synthetic pathway for cherimolacyclopeptide E (12)

$1672-1636 \mathrm{~cm}^{-1}$ (Amide I band) and $\mathrm{NH}$ bending at 1536-1522 $\mathrm{cm}^{-1}$ (Amide II band), confirming positivity of coupling reaction. Cyclization of linear peptide fragment 11 was supported by disappearance of absorption bands at $1750 \mathrm{~cm}^{-1}$ and $1390,1365 \mathrm{~cm}^{-1}(\mathrm{C}=\mathrm{O}$ stretching of ester and $\mathrm{CH}$ bending of tert-Butyl group) and presence of additional Amide I and Amide II bands of the -CO-NH- moiety at $1645-1642 \mathrm{~cm}^{-1}$ and $1521-1518 \mathrm{~cm}^{-1}$ in IR spectra of the compound 12. Formation of cyclopeptide was further confirmed by disappearance of signals at $157.5,79.5,53.3,29.2 \mathrm{ppm}$ corresponding to Boc and ester group in ${ }^{13} \mathrm{C}$ NMR spectrum and disappearance of singlets at 3.62 and $1.54 \mathrm{ppm}$ corresponding to three protons of methyl ester and nine protons of ${ }^{\text {B }}$ utyl group of Boc, in ${ }^{1} \mathrm{H}$ NMR spectrum of compound 12. Furthermore, ${ }^{1} \mathrm{H}$ NMR and ${ }^{13} \mathrm{C}$ NMR spectra of synthesized cyclic hexapeptide showed characteristic peaks confirming presence of all the 42 protons and 33 carbon atoms. Presence of $\left(\mathrm{M}^{+}+1\right)^{+}$ion peak (base peak) at $\mathrm{m} / \mathrm{z} 635.7$ corresponding to the molecular formula $\mathrm{C}_{33} \mathrm{H}_{42} \mathrm{~N}_{6} \mathrm{O}_{7}$ in mass spectra of compound 12, along with other fragment ion peaks resulting from cleavage at gly-phe amide bond level, showed exact sequence of attachment of all the six amino acid moieties in a chain. In addition, elemental analysis of compound $\mathbf{1 2}$ afforded values ( \pm 0.03 ) strictly in accordance to the molecular composition.

\section{PHARMACOLOGY}

Synthesized compound $\mathbf{1 2}$ was tested for in vitro antimicrobial, anthelmintic and cytotoxicity activites and results of biological activity studies are tabulated in Table 1, Table 2 and Table 3. 
Table 1: Antimicrobial activity data for compound 12.

\begin{tabular}{|c|c|c|c|c|c|c|c|c|}
\hline Compd. & \multicolumn{9}{|c|}{ Diameter of zone of inhibition (mm) } \\
\hline & \multicolumn{9}{|c|}{ Bacterial strains } & \multicolumn{4}{c|}{ Fungal strains } \\
\hline & $\begin{array}{c}B . \\
\text { subtilis }\end{array}$ & $\begin{array}{c}\text { S. } \\
\text { aureus }\end{array}$ & $\begin{array}{c}P . \\
\text { aeruginosa }\end{array}$ & $\begin{array}{c}\text { E. } \\
\text { coli }\end{array}$ & $\begin{array}{c}\text { C. } \\
\text { albicans }\end{array}$ & $\begin{array}{c}\text { M. } \\
\text { audouinii }\end{array}$ & $\begin{array}{c}\text { A. } \\
\text { niger }\end{array}$ & $\begin{array}{c}\text {. } \\
\text { mentagrophytes }\end{array}$ \\
\hline $\mathbf{1 2}$ & - & - & $28(6)^{\mathrm{a}}$ & $24(12.5)$ & $25(6)$ & $9(12.5)$ & - & $11(12.5)$ \\
\hline Control & - & - & - & - & - & - & - & - \\
\hline Ciprofloxacin & $20(6)$ & $20(12.5)$ & $25(6)$ & $19(12.5)$ & - & - & - & - \\
\hline Griseofulvin & - & - & - & - & $20(6)$ & $18(6)$ & $18(12.5)$ & $20(6)$ \\
\hline
\end{tabular}

${ }^{a}$ Values in parenthesis are MIC values $\left(\mu \mathrm{g} \mathrm{mL}^{-1}\right)$.

Table 2: Anthelmintic activity data for compound $\mathbf{1 2 .}$

\begin{tabular}{|c|c|c|c|c|c|c|}
\hline \multirow[b]{3}{*}{ Compd. } & \multicolumn{6}{|c|}{ Earthworm species } \\
\hline & \multicolumn{2}{|c|}{ M. konkanensis } & \multicolumn{2}{|c|}{ P. corethruses } & \multicolumn{2}{|c|}{ Eudrilus $s p$} \\
\hline & $\begin{array}{l}\text { Mean paralyzing } \\
\text { time }(\mathrm{min})^{\mathrm{a}}\end{array}$ & $\begin{array}{l}\text { Mean } \\
\text { death time } \\
(\min )^{\mathrm{a}}\end{array}$ & $\begin{array}{l}\text { Mean paralyzing } \\
\text { time (min) }\end{array}$ & $\begin{array}{l}\text { Mean } \\
\text { death time } \\
(\min )\end{array}$ & $\begin{array}{l}\text { Mean paralyzing } \\
\text { time (min) }\end{array}$ & $\begin{array}{l}\text { Mean } \\
\text { death time } \\
(\min )\end{array}$ \\
\hline $\begin{array}{l}\mathbf{1 2}^{\mathrm{b}} \\
\text { Control }^{\mathrm{c}} \\
\text { Mebendazole }^{\mathrm{b}} \\
\text { Piperazine citrate }^{\mathrm{b}}\end{array}$ & $\begin{array}{l}14.20 \pm 0.52 \\
- \\
10.55 \pm 0.64 \\
12.39 \pm 0.36\end{array}$ & $\begin{array}{l}17.32 \pm 0.47 \\
- \\
12.59 \pm 0.53 \\
13.52 \pm 0.49\end{array}$ & $\begin{array}{l}27.29 \pm 0.36 \\
- \\
17.58 \pm 1.03 \\
19.06 \pm 0.57\end{array}$ & $\begin{array}{l}29.32 \pm 0.57 \\
- \\
19.42 \pm 1.20 \\
22.23 \pm 0.78\end{array}$ & $\begin{array}{l}14.56 \pm 0.21 \\
- \\
11.35 \pm 0.45 \\
12.46 \pm 0.15\end{array}$ & $\begin{array}{l}17.05 \pm 0.11 \\
- \\
13.46 \pm 0.62 \\
13.58 \pm 0.47\end{array}$ \\
\hline
\end{tabular}

${ }^{\mathrm{a}}$ Data are given as mean \pm S.D. $(\mathrm{n}=3) ;{ }^{\mathrm{b}} c=2 \mathrm{mg} \mathrm{mL}^{-1} ;{ }^{\mathrm{c}} 0.5 \%$ Tween 80 in distilled water

Table 3: Cytotoxic activity data for compound $\mathbf{1 2}$.

\begin{tabular}{|c|c|c|c|c|c|c|c|c|c|}
\hline \multirow[t]{3}{*}{ Compd. } & \multirow{3}{*}{$\begin{array}{l}\text { Conc. } \\
(\mu \mathrm{g} / \mathrm{ml})\end{array}$} & \multicolumn{4}{|c|}{ DLA cells } & \multicolumn{4}{|c|}{ EAC cells } \\
\hline & & \multirow{2}{*}{$\begin{array}{l}\text { Live } \\
\text { cells } \\
\text { counted }\end{array}$} & \multirow{2}{*}{$\begin{array}{l}\text { No. of } \\
\text { dead } \\
\text { cells }\end{array}$} & \multirow{2}{*}{$\begin{array}{l}\% \\
\text { growth } \\
\text { inhibition }\end{array}$} & \multirow[t]{2}{*}{$\begin{array}{l}\mathrm{CTC}_{50}{ }^{\mathrm{b}} \\
(\mu \mathrm{M})\end{array}$} & \multirow{2}{*}{$\begin{array}{l}\begin{array}{l}\text { Live } \\
\text { cells }\end{array} \\
\text { counted }\end{array}$} & \multirow{2}{*}{$\begin{array}{l}\text { No. of } \\
\text { dead } \\
\text { cells }\end{array}$} & \multirow{2}{*}{$\begin{array}{l}\text { \% } \\
\text { growth } \\
\text { inhibition }\end{array}$} & \multirow[t]{2}{*}{$\begin{array}{c}\mathrm{CTC}_{50} \\
(\mu \mathrm{M})\end{array}$} \\
\hline & & & & & & & & & \\
\hline \multirow[t]{5}{*}{12} & 62.5 & 0 & 38 & 100.0 & & 0 & 28 & 100.0 & \\
\hline & 31.25 & 2 & 36 & 94.74 & & 3 & 25 & 89.29 & \\
\hline & 15.63 & 7 & 31 & 81.58 & 2.76 & 9 & 19 & 67.86 & 4.96 \\
\hline & 7.81 & 11 & 27 & 71.05 & & 14 & 14 & 50.00 & \\
\hline & 3.91 & 20 & 18 & 47.37 & & 24 & 4 & 14.29 & \\
\hline \multirow[t]{5}{*}{ Control } & 62.5 & 38 & 0 & - & & 28 & 0 & - & \\
\hline & 31.25 & 38 & 0 & - & & 28 & 0 & - & \\
\hline & 15.63 & 38 & 0 & - & - & 28 & 0 & - & - \\
\hline & 7.81 & 38 & 0 & - & & 28 & 0 & - & \\
\hline & 3.91 & 38 & 0 & - & & 28 & 0 & - & \\
\hline Standard & 62.5 & 0 & 38 & 100.0 & & 0 & 28 & 100.0 & \\
\hline \multirow[t]{4}{*}{ (5-FU) } & 31.25 & 0 & 38 & 100.0 & & 0 & 28 & 100.0 & \\
\hline & 15.63 & 10 & 28 & 73.68 & 37.36 & 11 & 17 & 60.71 & 90.55 \\
\hline & 7.81 & 13 & 25 & 65.79 & & 19 & 9 & 32.14 & \\
\hline & 3.91 & 22 & 16 & 42.11 & & 23 & 5 & 17.86 & \\
\hline
\end{tabular}

a $\%$ growth inhibition $=100-\left[\left\{\left(\mathrm{Cell}_{\text {total }}-\mathrm{Cell}_{\text {dead }}\right) \times 100\right\} / \mathrm{Cell}_{\text {total }}\right] ;{ }^{\mathrm{b}} \mathrm{CTC}_{50}=$ cytotoxic conc. inhibiting $50 \%$ of percentage growth

Investigation of antimicrobial activity revealed that synthesized cyclohexapeptide $\mathbf{1 2}$ possessed potent antifungal activity against pathogenic fungi Candida albicans and good antibacterial activity against gram negative bacteria. Compound $\mathbf{1 2}$ was almost $25 \%$ more active against pathogenic yeast $C$. albicans with MIC value of $6 \mu \mathrm{g} \mathrm{mL}^{-1}$, in comparison to reference drug griseofulvin and exhibited $12-26 \%$ more antibacterial activity against Pseudomonas aeruginosa and Escherichia coli with MIC value of 6 and 12.5 $\mu \mathrm{g} \mathrm{mL}{ }^{-1}$, in comparison to standard drug ciprofloxacin. Gram positive bacteria and fungus Aspergillus niger were found to be resistant to synthesized peptide.
However, compound $\mathbf{1 2}$ exhibited only slight antifungal activity against dermatophytes Microsporum audouinii and Trichophyton mentagrophytes.

Comparison of anthelmintic data indicated that compound $\mathbf{1 2}$ showed moderate anthelmintic activity against all three earthworms Megascoplex konkanensis, Pontoscotex corethruses and Eudrilus sp. in comparison to reference drugs mebendazole and piperazine citrate at concentration of $2 \mathrm{mg}$ $\mathrm{mL}^{-1}$. Pontoscotex corethruses was found to be the least sensitive earthworm species.

Moreover, compound 12 possessed high cytotoxic activity against DLA and 
EAC cell lines with CTC $_{50}$ values of 2.76 and $4.96 \mu \mathrm{M}$ respectively (Standard drug, 5-fluorouracil (5-FU): $\mathrm{CTC}_{50}$ values -37.36 and $90.55 \mu \mathrm{M}$ ), in comparison to significant cytotoxicity exhibited by natural cherimolacyclopeptide $\mathrm{E}$ against $\mathrm{KB}$ cells with $\mathrm{IC}_{50}$ value of $0.017 \mu \mathrm{M}$. On passing toxicity tests, synthesized cyclohexapeptide 12 may prove good candidate for clinical studies and can be new antimicrobial and cytotoxic drug of future.

\section{ACKNOWLEDGEMENTS}

The authors are grateful to U.S.I.C., DU, Delhi, India and R.S.I.C., I.I.T., Delhi, India for spectral analysis. Also, thanks to J.S.S.C.P., Ooty, India for carrying out cytotoxicity studies and C.P.C.R.I., Kasaragod, Kerala, India for providing earthworms for anthelmintic screening.

\section{REFERENCES}

1. M. T. Liu, S. Lin, Y. H. Wang, W. Y. He, S. Li, S. J. Wang, Y. C. Yang, J. G. Shi, Org. Lett., 9, 129 (2007)

2. P. W. Hsieh, F. R. Chang, C. C. Wu, K. Y. Wu, C. M. Li, S. L. Chen, Y. C. Wu, J. Nat. Prod., 67, $1522(2004)$

3. F. Abbas, R. Zayed, Z. Naturforsch. [C], 60, 813 (2005)

4. J. C. Luis, F. Valdes, R. Martin, A. J. Carmona, J. G. Diaz, Fitoterpia, 77, $469(2006)$

5. B. S. Min, N. Nakamura, H. Miyashiro, Y. H. Kim, M. Hattori, Chem. Pharm. Bull. (Tokyo), 48, 194 (2000)

6. A. Zamilpa, J. Tortoriello, V. Navarro, G. Delgado, L. Alvarez, Planta Med., 68, 281 (2002)

7. N. H. Tan, J. Zhou, Chem. Rev., 106, 840 (2006)

8. A. F. Morel, C. A. Araujo, U. F. da Silva, S. C. Hoelzel, R. Zachia, N. R. Bastos, Phytochemistry, 61, 561 (2002)

9. D. J. Craik, N. L. Daly, J. Mulvenna, M. R. Plan, M. Trabi, Curr. Protein Pept. Sci., 5, 297 (2004)

10. C. Jennings, J. West, C. Waine, D. Craik, M. Anderson, Proc. Natl. Acad. Sci. US A., 98, 10614 (2001)

11. W. Mongkolvisut, S. Sutthivaiyakit, H. Leutbecher, S. Mika, I. Klaiber, W. Moller, H. Rosner, U. Beifuss, J. Conrad, J. Nat. Prod., 69, 1435 (2006)
12. H. Morita, T. Kayashita, M. Shimomura, K. Takeya, H. Itokawa, Heterocycles, 43, 1279 (1996)

13. H. Morita, S. Nagashima, K. Takeya, H. Itokawa, Chem. Pharm. Bull. (Tokyo), 41, 992 (1993)

14. B. Picur, M. Cebrat, J. Zabrocki, I. Z. Siemion, J. Pept. Sci., 12, 569 (2006)

15. H. Morita, M. Eda, T. Iizuka, Y. Hirasawa, M. Sekiguchi, Y. S. Yun, H. Itokawa, K. Takeya, Bioorg. Med. Chem. Lett., 16, 4458 (2006)

16. H. Morita, T. Iizuka, C. Y. Choo, K. L. Chan, K. Takeya, J. Kobayashi, Bioorg. Med. Chem. Lett., 16, 4609 (2006)

17. H. Morita, T. Kayashita, H. Kobata, A. Gonda, K. Takeya, H. Itokawa, Tetrahedron, 50, 6797 (1994)

18. H. Morita, T. Kayashita, H. Kobata, A. Gonda, K. Takeya, H. Itokawa, Tetrahedron, 50, 9975 (1994)

19. Y. S. Yun, H. Morita, K. Takeya, H. Itokawa, J. Nat. Prod., 60, 216 (1997)

20. H. Itokawa, Y. Yun, H. Morita, K. Takeya, K. Yamada, Planta Med., 61, $561(1995)$

21. A. Wele, Y. Zhang, J-P. Brouard, J-L. Pousset, B. Bodo, Phytochemistry, 66, $2376(2005)$

22. R. Dahiya, D. Pathak, J. Serb. Chem. Soc., 72, 101 (2007)

23. R. Dahiya, Pak. J. Pharm. Sci., 20, 317 (2007)

24. R. Dahiya, D. Pathak, Asian. J. Chem., 19, 1499 (2007)

25. R. Dahiya, D. Pathak, J. Pharm. Res., 5, 69 (2006)

26. R. Dahiya, D. Pathak, M. Himaja, S. Bhatt, Acta Pharm., 56, 399 (2006)

27. R. Dahiya, D. Pathak, Egypt. Pharm. J. (NRC), 5, 189 (2006)

28. D. Pathak, R. Dahiya, J. Sci. Pharm., 4, 125 (2003)

29. M. Bodanszky, A. Bodanszky, The Practice of Peptide Synthesis, SpringerVerlag, New York, 1984.

30. A. W. Bauer, W. M. Kirby, J. C. Sherris, M. Turck, Am. J. Clin. Path., 45, $493(1966)$

31. L. C. Garg, C. K. Atal, Indian J. Pharm. Sci., 59, 240 (1963)

32. R. Kuttan, P. Bhanumathy, K. Nirmala, M. C. George, Cancer Lett., 29 , 197 (1985). 